\title{
LA CONCIENCIA PURA DEL TIEMPO Y EL MÉTODO ANALÍTICO EN LA «DEDUCCIÓN TRANSCENDENTAL DE LAS CATEGORÍAS (A)» DE KANT
}

\section{Eduardo N. Giovannini*}

\section{Introducción}

Un rasgo con el que usualmente se identifica la« Deducción Transcendental de las Categorías» de 1781 (A) ${ }^{1}$ consiste en destacar la permanente referencia que hace en ella Kant al carácter temporal de la conciencia humana. La apelación constante a lo largo de toda la argumentación a la forma temporal de nuestras representaciones sensibles es un elemento que distingue claramente a la primera de la segunda versión de la Deducción, de 1787. Ahora bien, la tendencia a destacar en todo momento el rol de la conciencia pura del tiempo en la constitución de los objetos de la experiencia ha sido tomada casi unánimemente como una evidencia del carácter "psicologista" que domina a la Deducción A . En efecto, ésta fue la lectura inmediata que se hizo del texto, tal como lo acredita la reseña de Garve-Feder, que ocasionaría incidentalmente la redacción de los Prolegómenos. Pero además ésta fue la lectura impulsada por corrientes disímiles, como el neokantismo de la Escuela de Marburgo, con Hermann Cohen ${ }^{2}$ como una figura destacada, y la tradición analítica, a través

* Becario de posgrado del Consejo Nacional de Investigaciones Científicas y Técnicas (CONICET). Recientemente ha publicado en libros y compilaciones de artículos, una serie de trabajos dedicados a la Deducción Transcendental de las Categorías y a la noción de intuición formal en la filosofía de la geometría de Kant. E-mail: engiovannini@gmail.com.

${ }^{1}$ Las referencias a la Crítica de la razón pura son dadas, como es habitual, de acuerdo a la paginación de la edición original. La primera edición (1781) se distingue con la letra (A), mientras que para la segunda edición (1787) se utiliza la letra (B). Citamos la Crítica según la edición de Mario Caimi: Kant, I., Crítica de la razón pura, Buenos Aires, Colihue, 2007.

${ }^{2}$ Cfr. Cohen, H., Kants Theorie der Erfabrung, Hildesheim, Georg Olms, 1987. 
de los comentarios pioneros de Peter Strawson ${ }^{3}$ y Jonathan Bennett ${ }^{4}$. La Deducción Transcendental de la segunda edición tiene así para estas corrientes una forma más consistente, en tanto que Kant consigue allí dirigir el argumento directamente al problema epistemológico, evitando de esa manera la doctrina anterior fundada en gran medida en la dudosa disciplina de la psicología transcendental.

Ahora bien, la centralidad que Kant le otorga a la actividad sintética de la imaginación, con sus distintas fases, se encuentra acompañada por otro rasgo determinante de la Deducción $\mathrm{A}$, a saber: la utilización del método analítico de exposición en casi la totalidad del texto. Este método es el que impone que se parta precisamente de los primeros elementos empíricos de nuestro conocimiento y se descubra en ellos, por medio de un argumento de forma regresiva, las condiciones de posibilidad en la que descansan. Empero, la relevancia de este rasgo a la hora de analizar la Deducción A no ha sido igualmente destacada, como sí lo ha sido la primacía de la conciencia pura del tiempo. Luego, el objetivo del presente artículo es analizar estos dos elementos que se presentan en la Deducción A. Sugeriremos que la importancia que Kant le atribuye al tiempo, o más precisamente, a la conciencia pura del tiempo, dentro de la argumentación de la Deducción A, no es solamente una consecuencia del espíritu psicologista que por esa época asoma en sus reflexiones. Más aún, defenderemos la tesis de que esta presencia es una consecuencia directa de la utilización del método analítico de exposición en la mayor parte del texto.

Para ello, en primer lugar presentaremos la distinción que traza Kant entre un método analítico y un método sintético de exposición. Seguidamente, analizaremos la forma que adquiere el argumento de la Deducción A cuando se sigue el método sintético, es decir, reconstruiremos el argumento dado en la "exposición desde arriba" de la Deducción Objetiva. Y finalmente indagaremos las diferencias notorias que este argumento sufre cuando se adopta, como en la mayoría de la Deducción A, el método inverso de exposición, i. e., el analítico.

${ }^{3}$ Cfr. Strawson, P., The bounds of sense: an essay on Kant's Critique of Pure Reason, London, Methuen, 1966.

${ }^{4}$ Cfr. Bennett, J., Kant's Analytic, London, Cambridge University Press, 1966. 


\section{El método analítico y el método sintético de exposición}

En todos los apartados de sus lecciones de lógica dedicados al tema del método, i. e., a la unidad de una totalidad de conocimientos de acuerdo a principios, Kant aborda sin excepción la cuestión de los distintos métodos de exposición que pueden ser seguidos para presentar una doctrina. Aunque no profundiza mucho el tema en estos escritos, Kant le atribuye una importancia considerable a la descripción de los distintos métodos posibles de exposición, en tanto que en ellos descasa la distinción lógica de los resultados de toda doctrina que intenten ser establecidos de manera apodíctica. Para ello, Kant distingue entre dos modos o métodos distintos de exposición, los cuales son denominados método analítico y método sintético. Las diferencias entre estos dos métodos las establece como sigue:

El método analítico se opone al sintético. Aquél comienza con lo condicionado y fundamentado y continúa hacia los principios (a principiatis ad principia). Éste, en cambio, va de los principios a las consecuencias o de lo simple a lo compuesto. El primero podría denominarse regresivo, el segundo progresivo ${ }^{5}$.

El método sintético consiste en tomar como punto de partida los principios y dirigirse desde allí hacia aquello que descansa en esos principios. El método analítico, en cambio, parte de lo condicionado, que es tomado como un hecho efectivo y real, y se remonta desde allí hasta su condición de posibilidad. Luego, el método sintético es el más perfecto, en tanto que no presupone nada, sino que demuestra la necesidad de cada paso que realiza y de cada elemento nuevo que introduce. Por el contrario, el método analítico parte necesariamente de una suposición, es decir, presupone que tenemos ya aquello que buscamos, y prosigue exhibiendo analíticamente los principios en los que reposa precisamente lo condicionado. De allí que, para Kant, el único método verdadero de exposición sea el método sintético.

${ }^{5}$ Logik, \$117, en Kant, I., Gesammelte Scbriften, herausgegeben von der Königlich Preussischen Akademie der Wissenschaften (en adelante Ak.), Berlín, Walter de Gruyter, 2da edición, 1968, Band. IX, p. 149. 
Por otro lado, el método analítico posee una ventaja por sobre el método sintético, a saber: su simplicidad y economía. El método analítico, señala el filósofo prusiano,

está siempre asociado a la popularidad, ya que uno se acostumbra a conocimientos abstractos al ascender hasta los principios, antes que habiendo comenzado por ellos. El método sintético es el más perfecto de todos, pero cuando me adapto a la capacidad de otros hombres, entonces comienzo con sus conceptos comunes, busco una regla basada en ellos, luego intento delinear un principio común, y así asciendo de los conocimientos más bajos hasta los más altos. Cuando uno ha ascendido, por medio del análisis, hasta los conocimientos abstractos, entonces puede conocerlos mucho más fácilmente in concreto, ya que sólo necesitamos servirnos de los conocimientos que hemos tomado previamente como fundamento ${ }^{6}$.

Un ejemplo del método analítico de exposición aparece en Prolegómenos a toda metafísica futura que baya de poder presentarse como ciencia $(1783)^{7}$. En efecto, lo que en este texto intenta ser demostrado, la proposición buscada, es la afirmación de que contamos con un conocimiento sintético, universal y necesario en la matemática y en las ciencias de la naturaleza. Ahora bien, en tanto que la argumentación sigue allí el método analítico de exposición, la forma que adquiere es la siguiente: se comienza suponiendo lo buscado como real y efectivamente existente, y se pasa luego a preguntarse ¿cómo son posibles la matemática pura y la ciencia pura de la naturaleza? Kant responde a esta pregunta mostrando que estos conocimientos descansan en, y son posibles por, las formas puras de la sensibilidad - espacio y tiempo - y los principios a priori de las síntesis llevadas a cabo por el entendimiento que se hallan pensadas en las categorías.

${ }^{6}$ Kant, I., The Heschel Logic, en M. Young (ed.), The Cambridge Edition of the Works of Immanuel Kant: Lectures on Logic, Cambridge, Cambridge University Press, 2004, p. 419.

${ }^{7}$ Cfr. Kant, I., Prolegómenos a toda metafísica futura que haya de poder presentarse como ciencia, en Ak. Band IV, p. 274. 
Luego, a primera vista, esta manera de justificar la afirmación resulta sin duda más directa y simple que el argumento que sigue la dirección inversa, es decir, que parte del principio de que las facultades del conocimiento humano son dos: sensibilidad y entendimiento, e intenta luego demostrar cómo el conocimiento en las ciencias antes mencionadas se funda en elementos que pertenecen a priori a estas facultades. El método analítico cuenta así con la ventaja de poder exhibir de un modo más fácil, simple y directo la conexión entre las proposiciones que se intentan demostrar y el o los principios en los que éstas descansan. Sin embargo, esta manera de proceder analíticamente se encuentra con la grave dificultad de quedar manifiestamente expuesta a la objeción escéptica. En efecto, si se llegase a dudar de la validez de aquello que es tomado como punto de partida de la exposición, y que es supuesto como efectivamente existente y verdadero (en este caso, existencia misma de la matemática y la ciencia pura de la naturaleza, en tanto conocimientos válidos a priori y universalmente), evidentemente el argumento debe ser abandonado y la justificación deberá buscar otro camino.

Veamos entonces cómo operan estos dos métodos de exposición en la estructura de la prueba de la Deducción A.

\section{La exposición "desde arriba" en la Deducción Objetiva}

En el Prefacio a la primera edición de la Crítica, Kant advierte que es posible distinguir dos caras o lados en el argumento llevado a cabo en la Deducción Transcendental (A). Los denomina, respectivamente, la "Deducción Subjetiva" y la "Deducción Objetiva". La Deducción Subjetiva se compone principalmente por la doctrina de la triple síntesis y su finalidad es "considerar el entendimiento puro mismo, atendiendo a la posibilidad de él y a las potencias cognoscitivas en las cuales él se basa"8. Luego, este lado de la Deducción A se propone indagar el aspecto subjetivo de la relación entre nuestro pensamiento y sus objetos, en el sentido en que busca establecer cuáles son aquellas facultades de la mente que hacen posible el conocimiento de objetos, o dicho más generalmente, que hacen posible el 
pensar mismo. Por el contrario, lo que pretende demostrar y justificar la Deducción Objetiva es cómo las categorías pueden referirse a priori a objetos, es decir, cómo las categorías, que son puramente intelectuales, son la condición de posibilidad de la unidad de la intuición sensible.

Como hemos dicho, la Deducción Objetiva tiene por fin explicar por qué sólo por medio de las categorías es posible que un objeto sea dado a la intuición. Ahora bien, el modo en que esta empresa es llevada a cabo también acepta una división en dos: la "exposición desde arriba" y la "exposición desde abajo". Tal división responde al punto de partida de la argumentación. En efecto, en la exposición desde arriba (A 116-119), Kant parte del "principio más alto de nuestro conocimiento", el principio de la apercepción transcendental, y avanza progresivamente hasta demostrar que todos los objetos de un conocimiento posible están referidos, por medio de las categorías, al entendimiento puro, lo cual determina que todos nuestros conocimientos tengan realidad objetiva. Por el contrario, en la exposición "desde abajo" (A 120-128), el orden de la argumentación es invertido. Partiendo de los elementos empíricos del conocimiento se remonta - por medio de un análisis regresivo - hasta las condiciones últimas de éste.

Ahora bien, la exposición "desde arriba" presenta rasgos similares a la posterior Deducción Transcendental según la segunda edición (B), en tanto toma como su punto de partida el principio de la apercepción transcendental. Esta es una evidencia, como ha sido notado, de la aplicación del método sintético. ${ }^{9}$ Es decir, el interés que presenta para nosotros esta versión del argumento es que es la única que, como veremos más adelante, sigue el método sintético de exposición en la Deducción A. Analicemos cuál es el recorrido del argumento.

Si bien la noción de apercepción transcendental, que ahora aparece como primer principio del que parte el argumento, ya ha sido caracterizada previamente por Kant en la Deducción Subjetiva, podemos decir que la

9 Para un excelente estudio de la relevancia del método sintético de exposición en la Deducción B, puede verse el reciente libro de Caimi, M., Legons sur Kant. La déduction transcendentale dans la deuxième édition de la Critique de la raison pure, París, Publications de la Sorbonne, 2007. 
exposición "desde arriba" comienza con una definición de esta noción: entiéndase por apercepción transcendental, señala Kant, a "la integral identidad de sí mismo a través de todas las representaciones posibles". ${ }^{10}$ Luego, partiendo de esta definición, a la cual se le añadirán más elementos en el transcurso de la argumentación, creemos que es posible reconstruir el argumento en cuatro tesis principales y una conclusión.

Tesis 1: Todas las intuiciones -en cuanto representaciones sensiblesestán sujetas a la condición de la unidad transcendental de la apercepción; y esa unidad, en tanto lo dado al sujeto es un múltiple, es sintética. ${ }^{11}$

Kant advierte en el inicio de la prueba que todas las intuiciones, en la medida en que no son sino representaciones sensibles, deben poder ser acogidas en la conciencia. Sin esta pertenencia de toda representación - en este caso de la intuición - a una conciencia, que se reconoce a sí misma como idéntica frente a la pluralidad de representaciones, éstas no serían nada. El acto que hace posible determinar la pertenencia de toda representación a un Yo único e idéntico es la apercepción pura. Luego, la apercepción transcendental está asociada a la integral identidad de sí mismo a través de todas las representaciones, es decir, a la posibilidad de reconocer que el yo al que le pertenecen todas las representaciones es uno y el mismo. $\mathrm{Y}$ es evidente que en esta condición descansa la posibilidad de todo conocimiento. La identidad de la conciencia se conforma a priori como el correlato de una pluralidad de representaciones, del mismo modo que éstas suponen a una conciencia única en donde puedan ser reunidas y enlazadas. A esta necesidad de que, para poder llegar a ser algo para nosotros, las representaciones tienen que poder ser reunidas en una única conciencia, y por lo tanto, llevadas a una unidad, la llama Kant principio transcendental de la unidad de todos los elementos diversos de nuestras representaciones. Mas, en tanto que no sólo esta conciencia de un yo único e idéntico puede únicamente ser alcanzada cuando una pluralidad de representaciones es

10 A 116.

${ }^{11}$ Cfr. A 116-117. 
enlazada en una conciencia, sino que además el contenido mismo de una intuición es un múltiple, la unidad que adquieren las representaciones al ser referidas al yo puro es sintética.

Ahora bien, toda unidad sintética presupone indefectiblemente una síntesis, que al ser necesaria, deber ser a priori. Pero toda síntesis de lo múltiple sensible es una tarea de la facultad de la imaginación, de donde se explica que:

Tesis 2: La apercepción transcendental, como unidad sintética, se refiere a la síntesis pura de la imaginación, como condición a priori de todo lo múltiple del conocimiento. ${ }^{12}$

Kant advierte que la síntesis de la imaginación, que tiene una referencia necesaria a la apercepción, reviste un carácter a priori. De allí que sólo puede ser pensada como una síntesis productiva y no como una reproductiva. Ahora bien, Kant aclara que "a la síntesis de lo múltiple la llamamos transcendental cuando, sin [hacer] diferencia de las intuiciones, se dirige a priori nada más que al mero enlace del múltiple"13. La síntesis productiva de la imaginación tiene por lo tanto la función de realizar el enlace de lo múltiple dado en las intuiciones, aunque independientemente del contenido de cada intuición en particular. La síntesis transcendental de la imaginación proporciona la unidad formal de toda síntesis sensible de las representaciones, y es por ello que es posible concluir que "la unidad transcendental de la síntesis de la imaginación es la forma pura de todo conocimiento posible"14.

Tesis 3: Sólo por medio de la unidad transcendental de la síntesis de la imaginación pueden ser representados los objetos de una experiencia posible.

${ }^{12}$ Cfr. A 118.

${ }^{13}$ A 118.

14 Ibid. 
Si recordamos que Kant había señalado un par de páginas antes que, "conocemos un objeto cuando hemos efectuado unidad sintética en el múltiple de la intuición"15, entonces esta tesis es una suerte de consecuencia de la tesis anterior. En efecto, si la unidad sintética necesaria de un múltiple en la intuición, que es lo que hace posible que una representación tenga validez objetiva, sólo puede ser lograda por medio de la síntesis transcendental de la imaginación, entonces sólo por medio de ésta pueden llegar a ser representados los objetos de una experiencia posible. Luego, para poder formular la cuarta tesis, Kant se apoya en la siguiente definición de la facultad del "entendimiento", definición que por otra parte se modificará sustancialmente en la segunda edición de la Deducción Transcendental en 1787: "La unidad de la apercepción con respecto a la síntesis de la imaginación es el entendimiento, y esa misma unidad, con respecto a la síntesis transcendental de la imaginación, [es] el entendimiento puro". ${ }^{16}$

Tesis 4a: En el entendimiento hay conocimientos puros a priori que contienen la unidad necesaria de la síntesis pura de la imaginación con respecto a todos los fenómenos posibles.

Por medio de esta tesis Kant no hace más que establecer el origen de los conceptos puros del entendimiento o categorías en el entendimiento puro. Sin embargo, en tanto que aquí el entendimiento es definido como la referencia de la apercepción transcendental a la síntesis pura de la imaginación, y no como una facultad independiente, es posible afirmar que, tal como resultan concebidas en la Deducción A, las categorías tienen desde su origen mismo una referencia inextricable a la síntesis transcendental de la imaginación. Ahora bien, esta síntesis, en tanto que tiene como fin conducirnos a un conocimiento objetivo, debe acontecer de acuerdo a reglas que deben ser necesariamente a priori. Existen, de esa manera, conocimientos en el entendimiento que contendrán la unidad necesaria de la síntesis pura de la imaginación. Estos conocimientos no son otros que las categorías. En las categorías se fundan todos los actos sintéticos de la 
imaginación, y por lo tanto, son las que hacen posibles nuestros conocimientos de los fenómenos.

Tesis 4b: El entendimiento, aunque por medio de la intuición de los objetos de los sentidos y de la síntesis de la imaginación, se refiere a todos los fenómenos de una experiencia posible.

El entendimiento puro, por medio de los conceptos puros o categorías que son su contenido, contiene en sí mismo, independientemente de cualquier materia dada, un principio formal y sintético para todos los fenómenos. De este modo, los fenómenos, para convertirse en un objeto de representación, deben poder conformarse a las categorías como las formas del pensamiento o como los principios formales de síntesis contenidas en la naturaleza misma del entendimiento. Sin esta necesaria referencia de los fenómenos al entendimiento, a través de las categorías, no podría nunca sernos presentado un objeto a la intuición. Y esta afirmación no es sino la conclusión del argumento:

Conclusión: El entendimiento, por medio de las categorías, es un principio formal y sintético de la experiencia, y los fenómenos están referidos necesariamente a él17.

Con esta afirmación Kant llega a probar la proposición buscada ${ }^{18}$.

${ }^{17}$ Cfr. Ibid.

18 Una reconstrucción alternativa de la "exposición desde arriba", pero que sin embargo no destaca la utilización del método sintético que aquí se hace, y por lo tanto, la diferencia fundamental con el resto de la Deducción A, puede verse en Barker, M., "The Proof Structure of Kant's A-Deduction", en Kant-Studien, vol. 92:2, 2001, pp. 259-282. 


\section{El método analítico y la conciencia pura del tiempo en la Deducción A}

El argumento que acabamos de analizar constituye más bien una excepción, cuando se lo compara con el esquema general de la prueba que se presenta en la Deducción A. Ello se debe a que el argumento "desde arriba" sigue el método sintético de exposición. Por el contrario, en el núcleo de la Deducción $\mathrm{A}$, es decir, la Deducción Subjetiva y la exposición "desde abajo" Kant apela al método analítico. Puede decirse que estas dos secciones son ciertamente las que distinguen a la primera versión de la Deducción, en tanto son desarrolladas in extenso. Es dable afirmar entonces que el método analítico parece ser un aspecto esencial de la misma. Pero veamos cuál es la forma de la argumentación cuando se utiliza este método.

Hemos indicado más arriba que el método analítico es un método regresivo, que impone que se parta de lo condicionado y se extraiga desde allí el principio de posibilidad del que aquello depende. En el caso de la Deducción A, lo condicionado, y por lo tanto, el punto de partida, está constituido por los primeros elementos empíricos del conocimiento, es decir, por la percepción. Aquellas exposiciones que sigan el método analítico, deberán partir entonces de la conciencia empírica del fenómeno, que es lo que Kant entiende por percepción. Ahora bien, la finalidad de la Deducción Transcendental consiste en poder llegar a justificar el empleo objetivo de los conceptos puros del entendimiento. Tal justificación, sin embargo, no puede alcanzarse directamente partiendo de una conciencia empírica dada y deduciendo o exhibiendo de allí los conceptos puros involucrados en ella, puesto que en ese caso lo que se conseguiría sería mostrar la validez de esos conceptos puros sólo para esa conciencia particular. La justificación buscada debe partir de una conciencia no empírica, que a su vez esté supuesta en toda conciencia empírica. Y esta conciencia a priori sólo puede ser la conciencia pura del tiempo.

No resulta casual que las secciones de la Deducción A donde se utiliza el método analítico - la Deducción Subjetiva y la exposición "desde abajo" - se inicien recordando una conclusión fundamental de la Estética Transcendental, a saber: la afirmación de que el tiempo es la forma universal 
de la sensibilidad ${ }^{19}$. Kant advierte que la percepción en su conjunto tiene $a$ priori por fundamento la intuición pura, y tomada ella como representación, su fundamento es la forma de la intuición interna, el tiempo. Kant parte así de la premisa de que el tiempo es la forma universal de la sensibilidad, y entiende la conciencia pura del tiempo como la conciencia más general de nuestra posibilidad de percibir, y con ello, de adquirir experiencia.

Toda percepción en tanto conciencia empírica del fenómeno supone por lo tanto, como su condición de posibilidad, la conciencia del tiempo. Kant se propone luego mostrar que nuestra representación del tiempo es el producto de una síntesis transcendental llevada a cabo de acuerdo a las categorías. Es decir, su estrategia consistirá en explicar cómo, a partir de la doctrina de la triple síntesis, al distinguir algunos caracteres obvios de la autoconciencia del tiempo, nos vemos conducidos a afirmar que ésta conlleva o envuelve actos que están regulados por conceptos puros. La triple síntesis se presenta así como la condición última de la intuición pura y, en tanto que ella determina a toda intuición empírica, se seguirá de allí que esta última está sometida a sus condiciones, i. e., a las categorías y a la apercepción transcendental como la condición última de toda síntesis.

No podemos detenernos aquí a analizar en detalle la doctrina de la triple síntesis, de modo que la caracterizaremos muy brevemente, para poder abordar el tema que nos interesa: su relación con la conciencia pura del tiempo.

Como hemos indicado más arriba, la doctrina de la triple síntesis se vuelve necesaria una vez que se pone de manifiesto un rasgo general que comparte toda conciencia intuitiva humana, a saber, su carácter temporal. Es decir, Kant había reconocido antes, en sección 10 de la Analítica Transcendental, una única síntesis de la imaginación ${ }^{20}$. Sin embargo, al comienzo de la Deducción A -tanto en la Deducción Subjetiva como en la exposición "desde abajo"- Kant toma como premisa fundamental la aseveración de que el tiempo es la forma universal de todos los fenómenos. Luego, este rasgo esencial de todas nuestras representaciones sensibles

\footnotetext{
${ }^{19}$ Cfr. A 99 y A 115.

${ }^{20}$ Cfr. B 102.
} 
parece hacer necesario ahora distinguir en la noción general de síntesis de la imaginación, tres momentos en función de los cuales esta síntesis es capaz de introducir unidad sobre la multiplicidad dada a la intuición.

Por otro lado, la importancia de reconocer el carácter temporal de la conciencia humana reside en que es precisamente éste el que determina que el contenido de la intuición no pueda ser nunca algo simple, sino que por el contrario siempre es una multiplicidad. Como lo señala Kant, "toda intuición contiene en sí un múltiple que, empero, no sería representado como tal, si la mente no pudiera distinguir el tiempo en la sucesión de las impresiones unas tras otras; pues en cuanto contenida en un instante, ninguna representación puede ser jamás otra cosa que absoluta unidad". ${ }^{21} \mathrm{La}$ posibilidad de que la intuición lo sea de algo múltiple reside en la capacidad de nuestra mente de distinguir el tiempo en la sucesión de las impresiones sensibles. El acto de intuir mismo debe ir acompañado de la conciencia de la sucesión temporal, de lo que se sigue que la intuición no es posible como un acto instantáneo, sino únicamente como un proceso. La intuición efectiva de una multiplicidad se funda así en la conciencia (pura) de la distinción del tiempo en la sucesión de las impresiones, puesto que toda representación contenida en un instante es una unidad absoluta: algo que para Kant no puede constituir un rasgo propio de nuestras representaciones intuitivas.

Del mismo modo, si el contenido de la intuición pura es siempre un múltiple -aunque de carácter puro- lo mismo valdrá para la intuición empírica, puesto que aquélla es condición de ésta. Ninguna intuición empírica puede ser así conciencia de algo simple, puesto que su objeto está condicionado por el objeto de la intuición pura -espacio o tiempo-. Ahora bien, esa multiplicidad pura no podría ser representada "como multiplicidad" en la intuición pura si yo no pudiera recorrer cada uno de sus elementos para reunirlos luego en una unidad, que nos permita tener una visión sinóptica de esa multiplicidad. La intuición pura -pero también la empírica- supone un acto puro que recorra, abarque y enlace lo múltiple dado en ella, acto que recibe el nombre de síntesis (pura) de la aprehensión. "Sin ella -declara Kant- no podríamos tener a priori ni las representaciones del 
espacio, ni [las] del tiempo; pues éstas sólo pueden ser generadas mediante la síntesis de lo múltiple que ofrece la sensibilidad en su receptividad originaria". 22 El carácter "activo" de toda síntesis pone así de manifiesto la participación necesaria de la espontaneidad de la mente en la génesis de la intuición pura del espacio y tiempo.

Mas lo aprehendido sucesivamente gracias a la síntesis de la aprehensión nunca podría dar lugar a una representación completa si no me fuera posible retener las impresiones que van pasando y se convierten en pasadas, y reunirlas luego con las presentes, por medio de su reproducción en la imaginación. Kant llama entonces a este acto que retiene lo pasado y lo asocia con lo presente, sintesis de la reproducción. ${ }^{23}$ De esta manera, la síntesis de la aprehensión está íntimamente ligada a la síntesis de la reproducción, y ambas son imprescindibles para que tenga lugar una intuición pura.

El paso siguiente consiste en mostrar que esta síntesis aprehensivoreproductiva sólo es posible por medio del empleo de conceptos que le dan unidad en lo que Kant identifica como la tercera fase, la sintesis del reconocimiento: "Sin conciencia de que lo que pensamos es precisamente lo mismo que lo que pensábamos un momento antes, sería inútil toda reproducción en la serie de las representaciones. Pues sería una representación nueva, en el estado actual, [representación] que no correspondería al acto mediante el cual ha tenido que ser generada poco a poco; y lo múltiple de ella nunca constituiría un todo, porque le faltaría la unidad que sólo la conciencia puede darle". ${ }^{24}$ Los conceptos son por lo tanto los encargados de proporcionar las reglas imprescindibles para que la actividad de síntesis produzca como resultado una unidad. $\mathrm{Y}$ en tanto que estas reglas, para que puedan llegar a producir un conocimiento, deben ser necesarias, estamos aquí en presencia de conceptos a priori.

Por último, la necesaria intervención de los conceptos que acontece en la síntesis del reconocimiento nos conduce al primer principio de todo nuestro conocimiento, y con ello, al fin del argumento, a saber: el principio

\footnotetext{
22 A $99-100$.

${ }^{23}$ Cfr. A 102.

${ }^{24}$ A 103.
} 
de la apercepción transcendental. La apercepción transcendental, considerada como la integral conciencia de sí mismo a través de todas las representaciones ${ }^{25}$, es la que hace posible la unidad de toda síntesis, y por lo tanto, en ella se funda la posibilidad de la síntesis que funda la intuición pura y la empírica. La posibilidad de que nuestro conocimiento empírico posea realidad objetiva, es decir, se refiera a objetos, se funda en la posibilidad de referir la multiplicidad fluyente de los fenómenos a la unidad de una conciencia autoconsciente por medio del acto de síntesis de las representaciones según reglas a priori, o sea, según las categorías. La representación de un objeto de una experiencia posible tiene como su condición última la apercepción, y por medio de ella, las categorías, que no son sino los conceptos fundamentales para pensar objetos en general que correspondan a los fenómenos. De esta manera, se llega a demostrar la validez objetiva de las categorías, que es precisamente lo que se pretendía probar.

Kant ha llegado a demostrar así la validez objetiva de las categorías, partiendo de la conciencia empírica, y tomando como "principio común" la conciencia pura del tiempo, como la conciencia a priori más general de la posibilidad de percibir. Ahora bien, si reparamos en las características del método analítico de exposición, podemos concluir enseguida que es precisamente éste el que impone que se parta de la conciencia del tiempo, y ello en tanto el aspecto puro que se puede observar más inmediatamente como la posibilidad de la conciencia empirica es precisamente la intuición pura del tiempo. Expliquémonos.

En el caso de la Deducción Transcendental (A), la proposición buscada es la que afirma que los conceptos puros del entendimiento valen $a$ priori para todos los objetos de una experiencia posible, mientras que el principio fundamental es el de la apercepción transcendental. Luego, en las partes en las que en la Deducción A se hace uso del método analítico, la argumentación debe partir de la intuición empírica del fenómeno, que acompañada de conciencia recibe el nombre de percepción. Mas es evidente que el aspecto puro más inmediato que podemos encontrar aquí es la 
conciencia pura del tiempo, en tanto que ella está en la base de todo proceso perceptual. Toda intuición de una multiplicidad, sea ésta empírica o pura, es posible gracias a la conciencia de las distintas fases de la sucesión temporal, en función de la cual es aprehendida la diversidad de impresiones. La conciencia a priori del tiempo revela así que la intuición pura del tiempo es el producto de una síntesis a priori en la que intervienen conceptos puros. Se establece de esta manera la necesidad de una triple síntesis como la condición de posibilidad de la intuición pura del tiempo (y del espacio). Exhibida la conexión entre la intuición empírica del fenómeno y la intuición pura del tiempo, producto de la triple síntesis, nos encontramos de ese modo frente al principio fundamental: la apercepción transcendental. La unidad de todo acto reside en última instancia en la apercepción pura, que es la conciencia de sí que permite reconocer la mismidad del proceso sintético. $\mathrm{Y}$ en tanto las categorías resultan definidas como las condiciones universales de la reunión de una multiplicidad intuitiva en una conciencia autoconsciente, i. e., como las reglas universales de la síntesis de representaciones, resulta lícito concluir que ellas son los conceptos fundamentales para pensar objetos en general para la intuición. Luego, se observa así que el hilo conductor que permite unir a la percepción de los fenómenos con el principio de la apercepción transcendental, y mediante esa unión justificar la aplicabilidad de las categorías a los objetos reales de la intuición empírica, es la conciencia pura del tiempo.

Por otro lado, consideramos que nuestra tesis de acuerdo a la cual el uso del método analítico de exposición es lo que determina que la Deducción A le otorgue a la conciencia pura del tiempo un papel fundamental, se vuelve aún más plausible cuando se observa lo que ocurre en el pasaje en dónde aquélla adopta el método sintético, es decir, en la "exposición desde arriba". Como vimos, se parte allí del principio de la apercepción transcendental que, de acuerdo a la formulación que aquí recibe, se resume en la necesidad de que todas las intuiciones, como representaciones sensibles, están sujetas a la condición de la unidad transcendental de la apercepción (Tesis 1). A continuación, Kant prosigue afirmando que al ser el contenido de una intuición siempre una multiplicidad, la unidad de ese mismo múltiple en el sujeto es necesariamente sintética; de donde se sigue que la apercepción transcendental está referida a la condición de toda síntesis, la síntesis pura de 
la imaginación (Tesis 2). La unidad transcendental de la síntesis de la imaginación es la forma pura de todo conocimiento, así como la apercepción pura es el fundamento de esa posibilidad. De lo que se sigue que sólo por esta síntesis pueden ser representados los objetos de una experiencia posible (Tesis 3). No es necesario de este modo hacer ninguna apelación al carácter temporal de esta síntesis, como así tampoco a las condiciones de su posibilidad en la conciencia pura del tiempo. Esta necesidad ha sido por completo erradicada de la argumentación desde que se decide partir de la apercepción transcendental, y tomar como una premisa que no necesita de mayor justificación al hecho de que toda intuición contiene efectivamente un múltiple.

Finalmente, bastará para dar por concluida la prueba, con mostrar cómo la unidad de la apercepción en relación a la síntesis de la imaginación es el entendimiento puro. Éste, en cuanto se halla en posesión de conocimiento puros -las categorías- que contienen la unidad necesaria de la síntesis de la imaginación, proporciona reglas universales de la síntesis de todos los fenómenos. Y ello es lo mismo que decir que el entendimiento, a partir de sus conceptos puros, se refiere a todos los fenómenos de una experiencia posible (Tesis $4 \mathrm{a}$ y $4 \mathrm{~b}$ ). Hemos llegado de este modo a la conclusión: los conceptos puros del entendimiento tienen a priori validez objetiva. La proposición buscada ha sido así alcanzada partiendo desde el principio fundamental de la apercepción transcendental, $y$ sin bacer ninguna referencia a la conciencia del tiempo.

\section{Observaciones finales}

Hemos intentado mostrar en lo precedente cómo la adopción por parte de Kant del método analítico de exposición, en la mayor parte del texto de la Deducción $\mathrm{A}$, tiene una incidencia directa en la atribución a la conciencia pura del tiempo de un rol vital en la estructura de la prueba. La estrategia seguida en esta versión de la Deducción coloca, desde su inicio mismo, la conciencia pura del tiempo como el principio fundamental que permite vincular la mera intuición empírica del fenómenos con síntesis de carácter transcendental, regladas por conceptos puros, y sometidas en última instancia al principio de la apercepción transcendental. De este modo, se refleja claramente en la estructura que adopta el argumento de la Deducción 
A ese rasgo que caracteriza el proceder del método analítico: partir de conceptos comunes, buscar una regla basada en ellos, delinear un principio común, para ascender de los conocimientos más bajos a los más altos. La conciencia pura del tiempo parece desempeñar así el rol de este principio común, que permite con más facilidad ligar a la afirmación que se pretende demostrar, con el principio más alto del cual ella depende.

Ahora bien, esta consecuencia que conlleva la preponderancia del método analítico en la Deducción A, nos lleva a preguntarnos acerca de si es lícito atribuirle exclusivamente a las aparentes inclinaciones psicologistas de Kant en esa época, la forma final y el rasgo característico que recibe la Deducción A, a saber: el hacer de la síntesis transcendental de la imaginación, con sus diversos momentos, el acto objetivamente fundamental que da lugar a la constitución de los objetos de la experiencia. Parecería más bien que el empleo del método analítico, con sus características de simplicidad, economía y popularidad, tiene algo que aportar al respecto. $\mathrm{Y}$ sin duda, esta cuestión resulta sumamente relevante a la hora de intentar una nueva lectura que se ocupe del problema de cómo efectivamente Kant juzgó con posterioridad las ideas presentadas en la Deducción A. Quizá este trabajo pueda servir como un punto de partida para comenzar a profundizar esta línea de interpretación.

\section{Resumen}

El objetivo del artículo es ofrecer una interpretación de la Deducción Transcendental de las Categorías (A) que ponga en relación dos de sus aspectos más distintivos y fundamentales: la referencia permanente al carácter temporal de la conciencia humana, y la utilización del método analítico de exposición. Se defenderá la tesis de que la conexión entre ambos aspectos es esencial, en tanto que es precisamente la adopción del método analítico lo que determina que se le confiera a la conciencia pura del tiempo un rol fundamental en la estructura del argumento. Con esta tesis se intenta ofrecer una lectura alternativa de la Deducción A, que se diferencie de la habitual según la cual la apelación constante de Kant a la representación pura del tiempo - y a las síntesis que lo hacen posible - es una consecuencia directa del carácter psicologista que la distingue.

Palabras clave: Kant; Deducción Transcendental (A); Conciencia pura del tiempo; Método analítico. 


\begin{abstract}
This paper aims to offer an interpretation of the Transcendental Deduction of the Categories (A) which puts together two of its most distinctive and fundamental traits: the constant reference to the temporal character of the human consciousness and the use of the analytic method of exposition. We will defend the thesis that the connection between both traits is essential, i. e., it will be argued that it is precisely the usage of the analytic method what confers to the pure consciousness of time a significant role in the structure of the argument. With this thesis an alternative reading of the A-Deduction is pursued, one which moves away from the predominant one which claims that Kant's persistent allusion in it to the pure time representation - and to the synthesis which make it possible - is just a direct consequence of its psychological character.
\end{abstract}

Key words: Kant; A-Transcendental Deduction; Pure consciousness of time; Analytic Method. 